\title{
A Quality Ratio-Based Novel Unequal Loss Protection Scheme in Wi-Fi Broadcasting System
}

\author{
Dong Hyun Kim, Jong Min Kong, and Jong Deok Kim
}

\begin{abstract}
The Wi-Fi broadcast system using the broadcast packet transmits the multimedia contents to many local mobile users in real time and it must use the FEC technology to recover the broadcast packet loss, because Media Access Control layer retransmission is not supported for broadcast packet loss. Equally adding the redundant packet is not very efficient due to the nature of the multimedia data. So, several studies have been made on the Unequal Loss Protection (ULP). Proposed ULP system can efficiently add the redundant packet to the source video data with experimentally modeling the deterioration of video according to the broadcast packet loss in Wi-Fi based broadcasting system. In order to predict the video deterioration, we use the Expected Quality Ratio (EQR). It is a ratio of the video deterioration of compression to the video deterioration of packet loss. And, we design the framework of ULP system to predict the video quality using the EQR. We implement and experiment the proposed algorithm. Experiment results show how our algorithm outperforms other recovery methods of packet loss.
\end{abstract}

Index Terms-Wi-Fi broadcasting system, video deterioration modeling, ULP, FEC.

\section{INTRODUCTION}

The Wi-Fi broadcast system involves a kind of mobile Internet Protocol Television (IPTV) technology that streams multimedia content to many local mobile users in real time. Unlike conventional Wi-Fi-based multimedia streaming systems that use a separate unicast packets to serve each user, the Wi-Fi broadcast system uses broadcast packets for scalability since one broadcast packet can serve many users simultaneously. However, it needs the packet recovery method for packet loss because Media Access Control (MAC) layer retransmission in not supported. Several studies have been made on efficient transmission for multimedia data in burst packet loss network. These studies can be divided into two main categories: One is application layer, and the other is transmission layer.

Video compression technology which reduces the video data size is developed in application layer, h.264 standard is representative. Packet loss recovery method is developed in transmission layer, the Automatic Repeat reQuest (ARQ) and Forward Error Correction (FEC) are representative. Because MAC layer retransmission is not supported in transmission of the Wi-Fi based broadcast packet, the Wi-Fi broadcast

Manuscript received November 10, 2012; revised January 28, 2013. This research was supported by Basic Science Research Program through the National Research Foundation of Korea (NRF) funded by the Ministry of Education, Science and Technology (2012-0001578).

The authors are with the Pusan National University, Pusan, 609-735, Republic of Korea (e-mail: dhkim1106@pusan.ac.kr, cocoriuse@mobile.re.kr, kimjd@pusan.ac.kr). system using broadcast packet has to use FEC method for packet loss recovery not the ARQ scheme [1].

By the way, because resources might be limited, applying the same FEC rate to all frames is not very efficient. Several studies have been made on improving the video quality with applying different FEC rate according to the characteristic of the multimedia frame. These are called Unequal Loss Protection (ULP) scheme [2].

Over the past few years, studies have focused on the ULP scheme. They analyzed the video coding characteristic which influences the video quality. The data units which can influence the decoded video quality are a set of packets in a stream, a set of macroblocks in a frame, a frame, and a video layer. The data units which determine the encoded video quality are macroblock ranking, frame classification, and video scalability exploitation [3]-[6].

Most of the proposed ULP schemes are effective at distortion minimization about packet loss or frame loss [7], [8]. Hartanto sets a fixed FEC rate to both intra and inter frame in proportion to the frame size. However, this method was not efficient because it did not reflect the influence about frame loss propagation in the GOP [9]. Yang analyzed the packet error distortion model in GOP and packet level. They found that video quality is proportional to Length Error Propagation. However, this method needs a large number of calculations because it uses the brute force search using LEP in order to reduce the reflection of packet loss in GOP [10]. Diaz proposed a video-aware ULP system for protecting RTP video streaming in busty packet loss network. They found that ULP system for RTP video streaming needs the new decision scope and new analysis scope. And, they proposed the DFS (Decision Frame Set) for frame protection and preferentially added redundant packet to the I-DFS for the I frame loss [11].

Several studies have been made on ULP using a video deterioration modeling about a packet loss. These studies generalize the video deterioration with a macroblocks unit in a frame about a GOP. An ULP studies based on video quality modeling use the PSNR as a criterion of video quality [12]. Considering the characteristics of wireless network, calculating the PSNR each frame is more efficient than other methods to find video quality but it needs a large number of calculations to find it. To overcome this problem, we model the video deterioration with experimental method and we use it for criterion of ULP algorithm.

The PSNR of received video does not fit for evaluating the video quality because it may differ from a quality of source videos about the same packet loss rate. Therefore, we propose the Quality Ratio $(\mathrm{QR})$ that can indicate the quality of received video about quality of sending video as criterion for performance evaluation. And, we propose the Expected 
Quality Ratio (EQR) which represents the video deterioration of frame within GOP by using probability and channel model. By predicting a received video quality in probability, the EQR proposes the ratio of FEC rate for the best video quality according to GOP size, packet loss rate, and redundant data rate.

\section{SYSTEM MODEL}

The system actors for the ULP system in Wi-Fi based broadcast system are an encoded video stream, a transmission channel and a FEC.

\section{A. Encoded Video Stream}

The multimedia source video data is encoded by video compression technology to efficiently transmit the multimedia video data. The H.264 standard is representative as video compression technology. However, because encoded video has a strong interrelationship between frames, the frame loss by packet loss causes negative effects on video quality. To overcome the deterioration in quality of video by packet loss, the H.264 limits the area of deterioration in quality to the GOP. The size of GOP and a coding rate determine the frame size and the video quality. When coding rate is $512 \mathrm{kbps}$, the quality of encoded video and the data size of encoded frame according to GOP size appear in Fig. 1[13].

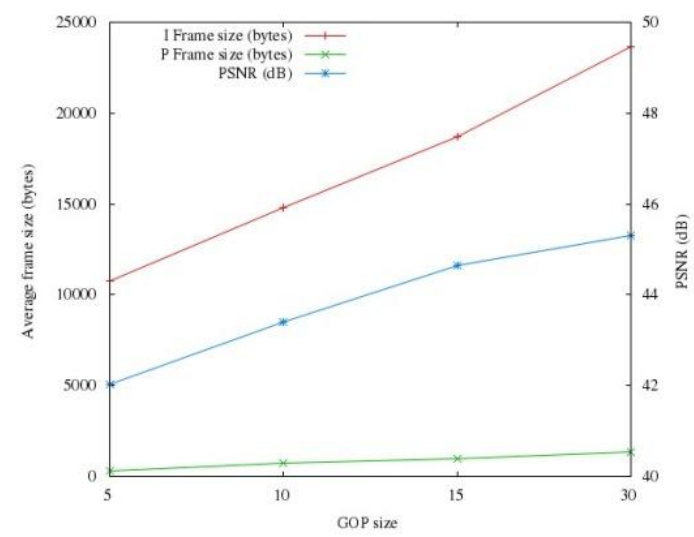

Fig. 1. The quality of encoded video and the data size of encoded frame according to GOP size, when a video coding is $512 \mathrm{kbps}$ (Akiyo).

\section{B. Transmission Channel}

In IP based networks, packet losses are of a bursty nature. In this paper, we use simplified Gibert-Elliot model to explain a bursty nature. To express the statistics characteristics in the communication channel, simplified Gibert-Elliot model uses the average loss rate $\left(P_{B}\right)$ and average burst loss rate $\left(L_{B}\right)$. Using the $P_{B}$ and $L_{B}$, the change probability is computed as shown in Eq. (1) [14].

$$
\begin{array}{r}
P_{g b}=\frac{p_{B}}{L_{B}\left(1-P_{B}\right)} \\
P_{b g}=\frac{1}{L_{B}}
\end{array}
$$

\section{FEC}

Fig. 2 shows the concept of FEC and the characteristic of encoded video streams. Encoding data frame is divided into many broadcast data packets for transmission. At this time, if one of the broadcast packets in whole broadcast packet is lost, the frame is lost. In this paper, we use the Reed Solomon code for recovery of packet loss. The $k$ of RS $(n, k)$ code refer to the number of data packets of a group and $n-k$ of $\operatorname{RS}(n, k)$ code refer to the number of redundant packets for the group.

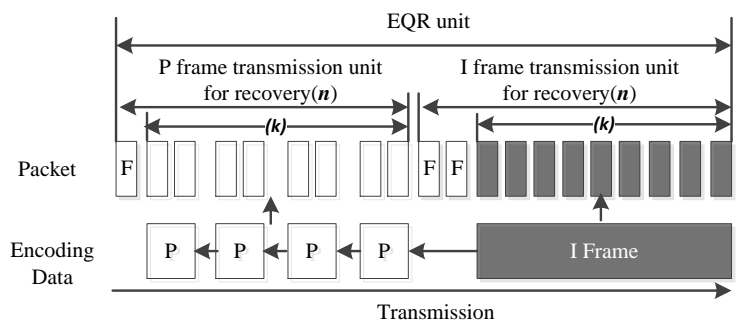

Fig. 2. The concept of FEC and characteristic of encoded video streams.

\section{PRotection Module}

Fig. 3 illustrates the framework of EQR based ULP system. The framework can be classified into three main parts: video coding, transmission of encoded video packet and calculation of EQR. The source video was encoded by the H.264 standard. At this time, a H.264 determines the GOP size and coding rate which can influence the video quality. Encoded video data for transmission is divided into encoded video packets according to the frame unit. And, ULP system calculates the Expected Quality Ratio (EQR) by using packet loss rate, available redundant data and GOP size. And, FEC packet assembler based on calculated EQR adds the redundant packet to encoded video packet and it transmits the encoded video packet to users.

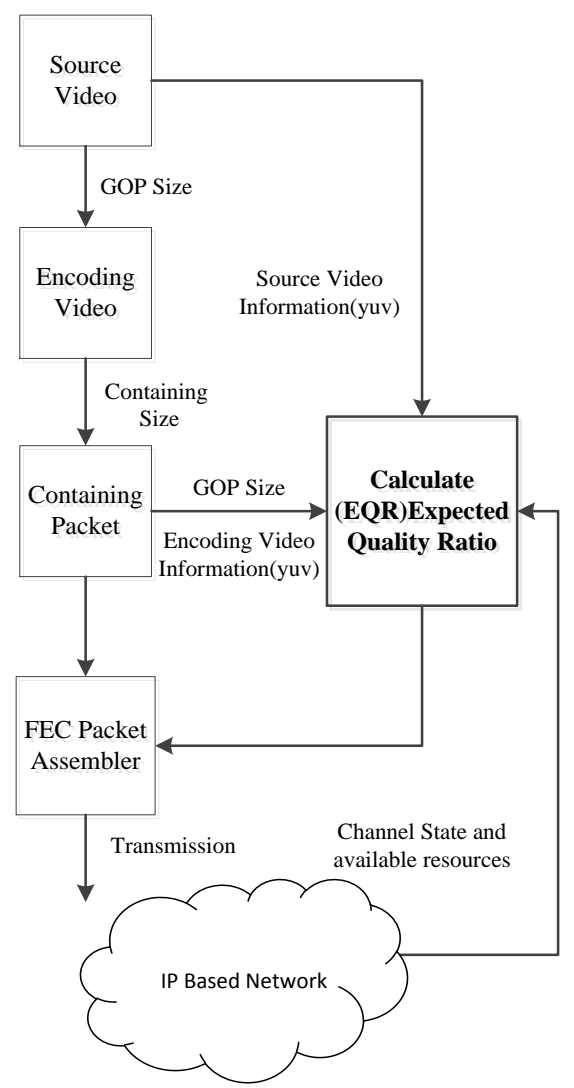

Fig. 3. EQR based ULP system framework. 


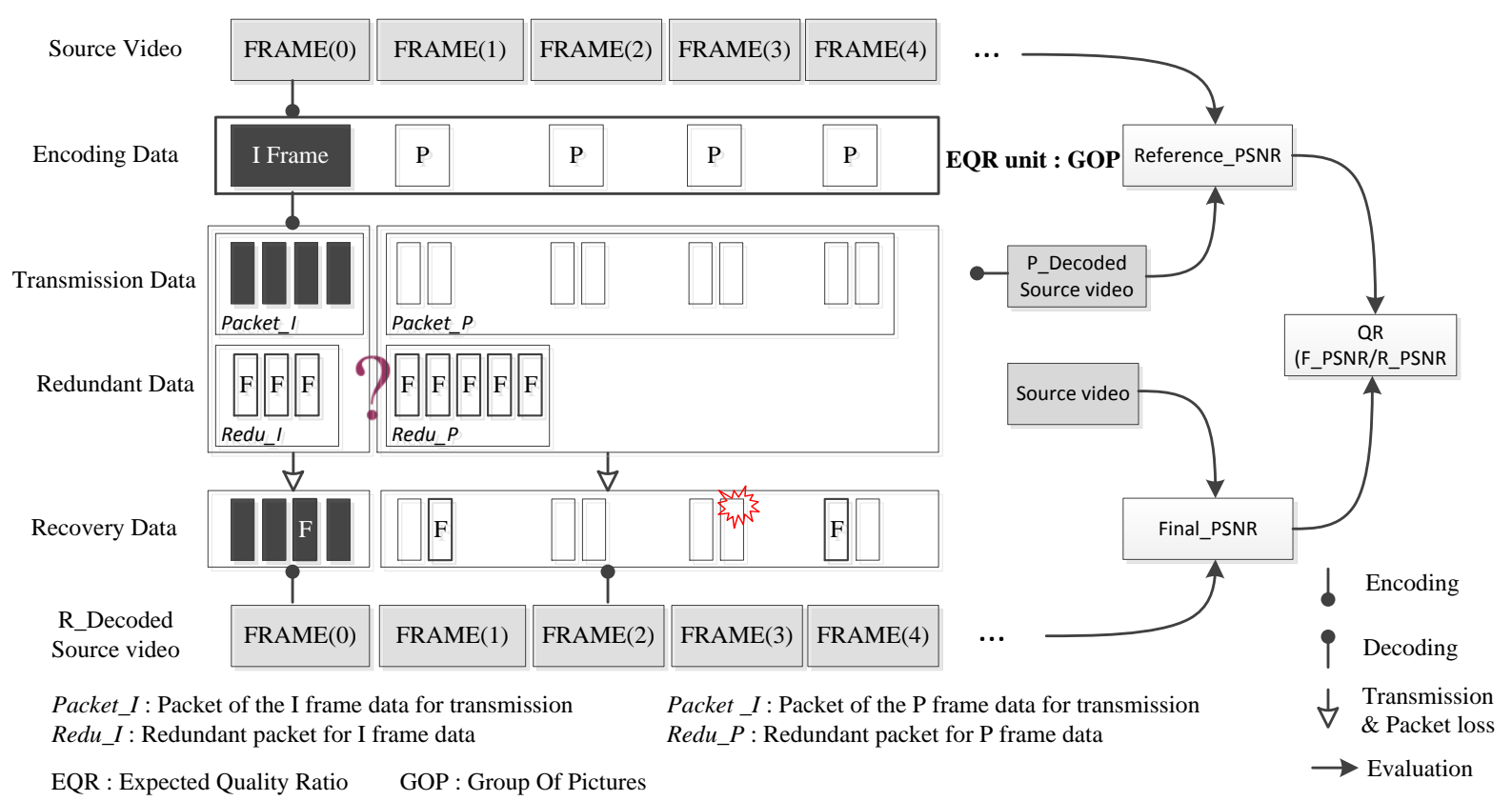

Fig. 4. The concept of $\mathrm{QR}$.

\section{CONCEPT OF QR AND VIDEO DETERIORATION MODEL}

\section{A. The Concept of $Q R$}

Fig. 4 illustrates the concept of QR for ULP system. The $\mathrm{QR}$ is a ratio of the video deterioration of compression to the video deterioration of packet loss. Source video is not encoded video source data. Then, source video data encoded by the H.264 technology and an encoded video data consists of three type of frames. Then, encoded frame is divided into packet for transmission in Wi-Fi broadcast system. In order to confirm the video deterioration by compression, we calculate the reference PSNR comparing with source video data and packetized video data. And, we transmit the packetized video data which adds the redundant packet to recover the packet loss. We calculate the final PSNR comparing with recovered video data and source video data. We define 'QR' as the ratio of reference PSNR to final PSNR and Equation is as follow.

$$
Q R=\frac{\text { reference _PSNR }}{\text { final_PSNR }}(0<Q R \leq 1)
$$

\section{B. The Video Deterioration Model}

Fig. 5 shows the characteristic of QR value according to loss or success of I frame in the GOP. H.264 has a strong dependence at I frame in source video encoding process. Then, through the experiment, we analyzed the characteristic of QR in the GOP according to loss or success of I frame.

\section{1) Reception success of I frame}

Given that a QR about I frame is QR (I), the QR (I) is 1 because the reference PSNR is equal to final PSNR. Let us assume that the $\mathrm{QR}$ about $k$ th $\mathrm{p}$ frame after a reception success of I frame is QR $(k)$.

$$
Q R(k)=\left(Q R(I)-C_{1}\right)^{n}
$$

In the I frame success of Fig. 5, the QR of a reception success of $\mathrm{P}$ frame is equal to a $\mathrm{QR}$ of previous frame. And, the $\mathrm{QR}$ of a reception failure represents the product of previous QR and C1. In case of I frame success, $k$ th $\mathrm{QR}$ can appear in Eq. (4) where $n$ is the number of $p$ frame loss.

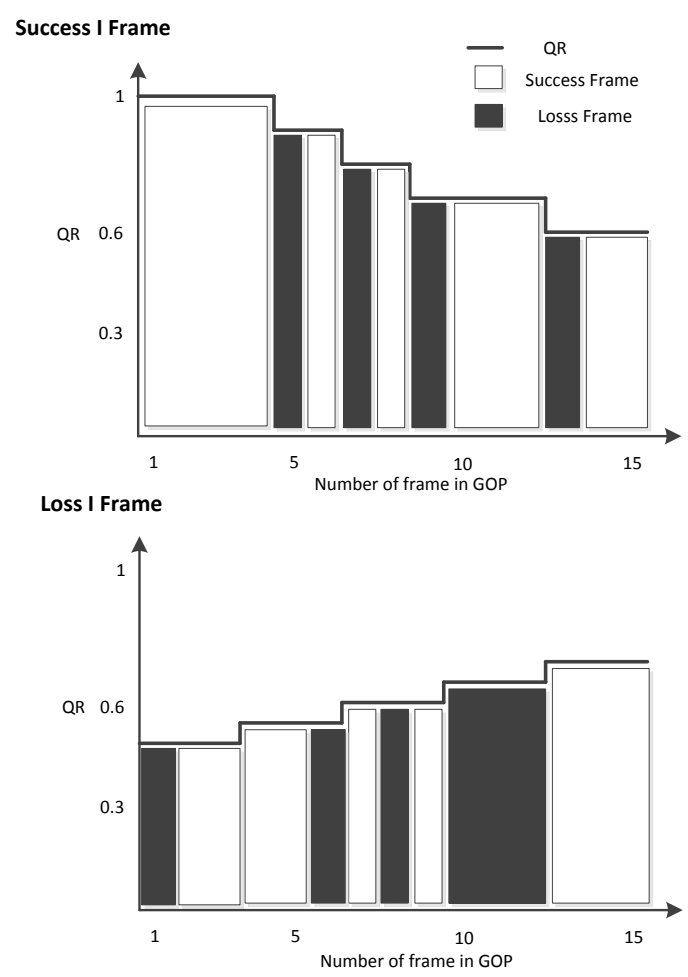

Fig. 5. QR according to I frame loss in GOP.

\section{2) Reception failure of I frame}

In case of the reception failure of I frame, all of frames in GOP decoded by the same frame. This frame is whether the last frame in previous GOP or the first frame in next GOP. Therefore, the QR of received video is stepped shape like Fig. 5. Because it is difficult to predict the QR in I frame loss, we use the average value of $\mathrm{QR}$ in GOP. The $\mathrm{C} 2$ refer to the Average Quality Ration (AQR) in reception failure of $\mathrm{I}$ frame. 


\section{3) Calculate $E Q R$}

Assume that a receiver suffers from the same loss probability $P$ in receiving each broadcast packet. That is, the receiver can successfully receive a packet with a probability $(1-P)$. With FEC, loss probability in I frame can get all data packets only if it can receive $k$ or less packets out of $n$ packets. Using the binomial theorem, we get I frame loss probability $E_{I}$ as shown in Eq. (4).

$$
E_{I}=\sum_{k=0}^{k-1}\left(\begin{array}{l}
n \\
k
\end{array}\right)(1-P)^{k} p^{n-k}
$$

Average Quality Ratio (AQR) refers to the average QR about all frames in GOP. And, we calculate the pattern occurrence probability (PCP) by using the Eq. (4) according to the distribution of redundant packet in GOP. Example, if the size of GOP is 5, 32 patterns happen. The EQR can represent the Eq. (5).

$$
E Q R=\sum_{k=1}^{2^{P}}(A Q R(k) \times(P C P(k))
$$

where the $p$ is GOP size and the $k$ is case of pattern occurrence.

\section{EXPERIMENT AND PERFORMANCE EVALUATION}

To evaluate the video quality, we use the Evalvid [15]. The video source and parameter for experiment appears in Table I.

Fig. 6 show the video quality of each packet recovery method in Akiyo video source and Fig. 7 shows the video quality of each packet recovery method in Foreman video source. The more packet loss increases, the less QR decreases because we use the limited redundant data $(64 \mathrm{kbps})$.

Although the Foreman video source and the Akiyo video source encode the same coding rate, a Foreman video source has less QR than the Akiyo video source because an encoded Video frame having different size of frame not recovered.

TABLE I: THE EXPERIMENT PARAMETER

\begin{tabular}{ll}
\hline \hline Parameter & Value \\
\hline Video source name & Akiyo, Foreman \\
FPS & 30 \\
Resolution & CIF(352X288) \\
Coding rate & $512 \mathrm{kbps}$ \\
GOP size & 15 \\
Packet loss rate & $0 \% \sim 25 \%$ \\
Redundant rate(packets/sec) & $64 \mathrm{kbps}(8$ packets/sec $)$ \\
Packet size & $1024 \mathrm{Byste}$ \\
\hline \hline
\end{tabular}

To evaluate the performance of proposed algorithm about video properties, we use the Akiyo and Foreman. The Akiyo has a similar frame size in each GOP because it has a low picture change, but the foreman is not. The size of redundant data is $64 \mathrm{kbps}$, the packet loss rate is from $0 \%$ to $25 \%$.

The experiment results for average $\mathrm{QR}$ are provided in Fig. 6 and Fig. 7. It can be observed that the proposed QR based ULP outperforms both the ELP and the hartanto method. The not recovery does not use the recovery packet. Then, it dramatically deteriorates the video quality with low packet loss rate. The ELP equally assigns the redundant packet to video packet in order to recover the packet loss.

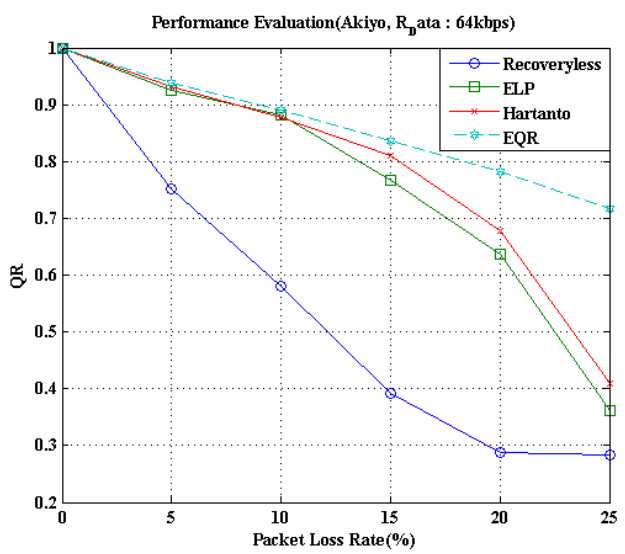

Fig. 6. Comparing the QR of packet recovery methods in Akiyo video.

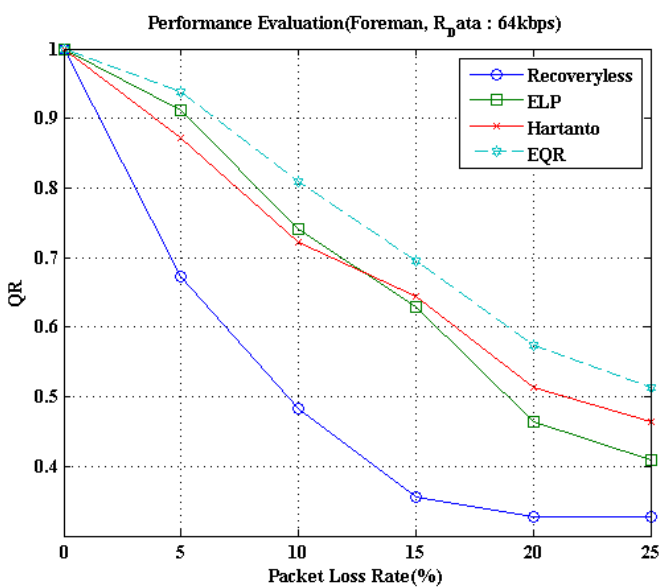

Fig. 7. Comparing the QR of packet recovery methods in Foreman video.

The more a packet loss rate increases, the more a video quality dramatically deteriorates because it does not consider the influence of video quality about packet loss. Hartanto sets a fixed FEC rate to both intra and inter frame in proportion to the frame size. However, this method was not efficient because it was not reflected the influence about frame loss propagation in the GOP.

\section{CONCLUSION}

A ULP system efficiently transmits the multimedia video data with the characteristic of a video deterioration. The PSNR of received video does not fit for evaluating the video quality because it may differ from a quality of source videos about the same packet loss rate. Then, we proposed the QR based ULP system. Proposed ULP system is more effective than an existing PNSR because it use a ratio of the video deterioration of compression to the video deterioration of packet loss. Then, we proposed the video deterioration model about frame loss and improved the video quality by predicting the video deterioration with a probability in GOP. As a performance evaluation, proposed ULP scheme improved the QR of approximately 0.34 than that of ELP and approximately 0.31 than that of the Hartanto method at packet loss of $25 \%$ in the Akiyo video and proposed ULP scheme improved the QR of approximately 0.1 than that of ELP and approximately 0.05 than that of the Hartanto method 
at packet loss of $25 \%$ in the Akiyo video.

\section{REFERENCES}

[1] A. Nafaa, T. Taleb, and L. Murphy, "Forward error correction strategies for media streaming over wireless networks," IEEE Communications Magazine, vol. 46, no. 1, pp. 72, January 2008.

[2] A. E. Mohr, E. A. Riskin, and R. E. Ladner, "Unequal loss protection graceful degradation of image quality over packet erasure channels through forward error correction," IEEE Journal on Selected Areas in Communications, vol. 18, no. 6, pp. 819-828, June 2000.

[3] B. Cavusoglu, D. Schonfeld, R. Ansari, and D. K. Bal, "Real-time lowcomplexity adaptive approach for enhanced QoS and error resilience in MPEG-2 video transport over RTP networks," IEEE Transactions on Circuits and Systems for Video Technology, vol. 15, no. 12, pp. 1604-1614 , Dec. 2005.

[4] F. Marx and J. Farah, "A novel approach to achieve unequal error protection for video transmission over 3G wireless networks," Signal Processing: Image Communication, vol. 19, no. 4, pp. 313-323, April 2004.

[5] Y. Chang, S. Lee, and R. Komyia, "A fast forward error correction allocation algorithm for unequal error protection of video transmission over wireless channels," IEEE Transactions on Consumer Electronics, vol. 54, no. 3, pp. 1066-1073, August 2008.

[6] H. Ha and C. Yim, "Layer-weighted unequal error protection for scalable video coding extension of H.264/AVC," IEEE Transactions on Consumer Electronics, vol. 54, no. 2, pp. 736-744, May 2008.

[7] W. Wen, H. Hsiao, and J. Yu, "Dynamic FEC-distortion optimization for H.264 scalable video streaming," IEEE 9th Workshop on Multimedia Signal Processing, 2007. MMSP 2007, no. 1-3, pp. 147-150, Oct. 2007.

[8] Y. Shi, W. Chengke, and D. Jianchao, "A novel unequal loss protection approaches for scalable video streaming over wireless networks," IEEE Transactions on Consumer Electronics, vol. 53, no. 2, pp 363-368, May 2007.

[9] F. Hartanto and H. R. Sirisena, "Hybrid error control mechanism for video transmission in the wireless IP networks," in Proc. selected Papers of 10th IEEE Workshop on Local and Metropolitan Area Networks, 2001, pp. 126-132.

[10] X. Yang, C. Zhu, Z. G. Li, X. Lin, and N. Ling, "An unequal packet loss resilience scheme for video over the Internet," IEEE Trans. Multimedia, vol. 7, no. 4, pp. 753-765, 2005.

[11] C. Diaz, J. Cabrera, F. Jaureguizar, and N. Garcia, "A video-aware FEC-based unequal loss protection system for video streaming over RTP," IEEE Transactions on Consumer Electronics, vol. 57, no. 2, pp. 523-531, May 2011.

[12] Y. Zhang, S. Qin, and Z. He, "Fine-Granularity Transmission Distortion Modeling for Video Packet Scheduling over Mesh
Networks," IEEE Transactions on Multimedia, vol. 12, no. 1, pp. 1-12, Jan. 2010.

[13] P. Pérez, J. Macías, J. J. Ruiz, and N. García, "Effect of Packet Loss in Video Quality of Experience," Bell Labs Technical Journal, vol. 16, no. 1 , June 2011.

[14] ITU-T Rec. G.1050, Network model for evaluating multimedia transmission performance over internet protocol, Nov. 2007.

[15] J. Klaue, B. Rathke, and A. Wolisz, "Evalvid - A Framework for Video Transmission and Quality Evaluation," presented at $13^{\text {th }}$ International Conference on Modelling Techniques and Tools for Computer Performance Evaluation, Urbana, Illinois, USA, September 2003.

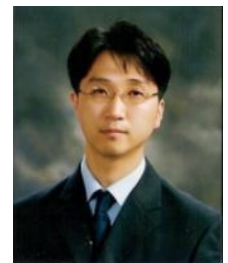

Dong Hyun Kim received his B.S. M.S. degrees in Information and Communication Engineering from Dong-Eui University in 1998 and 2004, respectively. $\mathrm{He}$ is now a Ph.D. candidate of Computer Science and Engineering in Pusan National University. His current research interests include RFID/USN, quality of services and mobility support in wireless networks.

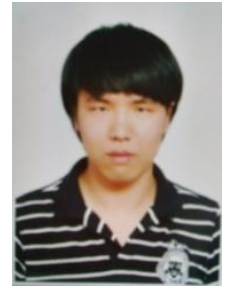

Jong Min Kong received his B.S. degrees in Information and Computer Engineering from Pusan National University in 2012. He is now a Postgraduate of Computer Science and Engineering in Pusan National University. His current research interests include RFID/USN, quality of services and mobility support in wireless networks.

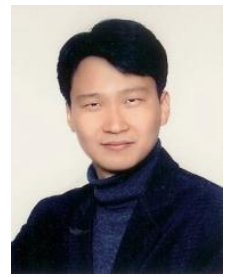

Jong Deok Kim received his B.S. M.S. degrees in Computer Science from Seoul National University in 1994 and 1996, respectively, his Ph. D. in Computer Science and Engineering from Seoul National University in 2003. He joined Telcoware in 2003, which is a Korea venture company focusing on wireless core network. $\mathrm{He}$ is now an Associate Professor of Computer Science and Engineering in Pusan National University since 2006. His current research interests include RFID/USN, quality of services and mobility support and open service architecture in wireless networks. 